\title{
Controlled study of metoprolol and propranolol during prolonged administration in patients with essential tremor
}

\author{
S CALZETTI, ${ }^{*}$ LJ FINDLEY,${ }^{*}$ E PERUCCA,$\dagger$ A RICHENS $\ddagger$ \\ From the Medical Research Council, Hearing and Balance Unit, The National Hospital, Queen Square, \\ London, * Istituto di Farmacologia Medica, Università di Pavia, Pavia, Italy † and the Department of \\ Pharmacology and Therapeutics, Welsh National School of Medicine, Cardiff, Wales $\ddagger$
}

SUMMARY The efficacy of propranolol and metoprolol in the treatment of essential tremor was compared in a double blind crossover placebo-controlled study in 16 patients. Each treatment was given for a period of 4 weeks at two different dosage regimens (150 and $300 \mathrm{mg}$ daily for metoprolol, 120 and $240 \mathrm{mg}$ daily for propranolol). Each dosage regimen lasted for 2 weeks. Tremor assessment was carried out by accelerometry, clinical evaluation, patient's self-rating and a battery of performance tests. At the lower dosage, propranolol was found to be superior to placebo on the basis of performance tests and patient's self-assessment. At the higher dosage, propranolol was superior to placebo on all methods of assessment. By contrast, the tremorolytic effect of metoprolol was not significantly different from that of placebo, irrespective of the dosage or of the method of assessment used. Propranolol (120 mg daily) was better than metoprolol ( $150 \mathrm{mg}$ daily) on the basis of clinical evaluation and patient's self-assessment. Propranolol ( $240 \mathrm{mg}$ daily) was superior to metoprolol (300 mg daily) on the basis of patient's self-assessment. Both drugs antagonised standing tachycardia to a similar extent. These results indicate that the effectiveness of metoprolol, previously demonstrated in a single-dose study in the same patients, is not fully maintained during prolonged administration. In the absence of specific contraindications, propranolol represents a better choice in the treatment of patients with essential tremor.

The role of $\beta_{1}$-selective adrenoceptor antagonists in the symptomatic relief of essential tremor has not been yet clearly established. Following initial reports claiming that metoprolol is effective, ${ }^{1-5}$ the controlled studies conducted so far with either metoprolol or atenolol have led to conflicting results. $^{6-8}$ In some of these studies, however, $\beta_{1}$-selective drugs have been compared with non selective $\beta$-adrenoceptor antagonists such as propranolol and sotalol at dosages not having equipotent $\beta_{1}$-blocking activity, thus making it difficult to obtain a quantitative assessment of their comparative tremorolytic potencies. This issue has important therapeutic implications because $\beta_{1}$ selective drugs may represent an alternative to

Address for reprint requests: Dr Stefano Calzetti, Istituto di Clinica Neurologica, Via del Quartiere 4, 43100 Parma, Italy.

Received 27 March 1982. Accepted 19 May 1982. propranolol in patients with essential tremor and obstructive disease of the upper airways. In a previous study ${ }^{9}$ we found that a single oral dose of metoprolol $(150 \mathrm{mg})$ reduced the amplitude of essential tremor to virtually the same extent as a $\beta_{1}$-equipotent dose of propranolol $(120 \mathrm{mg})$. This encouraging observation led us to compare the efficacy of these two drugs following prolonged administration in the same population of patients.

\section{Methods}

\section{Patients}

Sixteen patients affected by moderate to severe essential tremor (eight male and eight female) aged between 20 and 72 years (mean age 43 years) and attending the out patient clinics at the National Hospital for Nervous Diseases, Queen Square, gave their informed consent to participate in the study.

The diagnosis was established on the basis of the clinical history and detailed general and neurological examination accompanied by ancillary laboratory investigations, where 893 
necessary. All patients had been symptomatic for at least 1 year (range 1 to 43 years) prior to the study. In nine patients there was a family history of tremor affecting hands or head or both. Fifteen patients had previously taken part in a single oral dose study with the same drugs. ${ }^{9}$ No patient was receiving any drug therapy for tremor at the time of the study but nine of them had been previously treated with propranolol (range 80-240 mg daily). Patients with a history of excessive alcohol intake, congestive heart failure, A-V block, diabetes mellitus and asthma were excluded.

\section{Protocol}

The study was double-blind and consisted of three treatments (metoprolol, propranolol and placebo) arranged according to a crossover design. Each treatment lasted for four weeks. The active treatments consisted of two consecutive periods (each lasting for two weeks) during which two dosage regimens were used: 120 and $240 \mathrm{mg}$ (as 40 or $80 \mathrm{mg}$ tablets) daily for propranolol and 150 and 300 $\mathrm{mg}$ (as 50 or $100 \mathrm{mg}$ tablets) daily for metoprolol. These doses are considered, on the basis of literature data, to produce a comparable degree of cardiac $\beta$-blockade. ${ }^{10}$ The order of treatment was randomised, but within each active treatment period the lower dosage always preceded the higher dosage regimen. Drug (or placebo) were given in three equally divided daily doses. All tablets looked alike and the same number of tablets was taken in any one day of the study.

Assessment of hand tremor was performed at the end of each dosage period (that is every two weeks) as near as possible at the same time of day for each patient. Patients were instructed to take the previous dose about 2 hours before testing. Tremor was measured by means of piezoresistive linear accelerometers (ENDEVCO 7265-10) attached to the dorsal surface of the outstretched hands, the forearms being supported up to the wrists. ${ }^{9}$ On each recording session three separate tremor recordings of about 3 min duration were obtained at 5 min intervals. The signal was amplified, recorded on paper and stored on magnetic tape for off-line spectrum analysis. The following parameters were determined: dominant peak frequency $(\mathrm{Hz})$, acceleration $(\mathrm{g})$ and amplitude of hand displacement (mm). Only the data of the more severely affected hand were used for the computation of results. Details of the recording and analysis procedures have been described elsewhere. ${ }^{9}$

At the completion of the accelerometric recordings, clinical evaluation of the postural tremor of the more involved hand (held in the same position used for accelerometric recording) was carried out. Additional evaluations included the patient's self-assessment of tremor severity during daily activities and tests of performance (hand-writing, drawing geometrical figures and tracing an Archimede's spiral). A score from 0 to 5 (maximum of tremor severity) was used for the rating of each parameter. For the tests of performance, the mean score derived from the rating of three independent investigators was used for the statistical analysis.

Measurements of pulse rate were obtained after a $10 \mathrm{~min}$ rest in the supine position and repeated after 1,2 and $3 \mathrm{~min}$ of standing (the mean of these three values was used for the analysis). The inhibition of standing tachycardia was used as an approximate indication of cardiac $\beta$-blockade. "

Venous blood samples for the determination of serum propranolol or metoprolol levels were taken at the completion of the test. All tests were performed by the same investigator.

\section{Serum propranolol and metoprolol assay}

Serum propranolol and metoprolol concentrations were measured by high pressure liquid chromatography according to a modification of the method of Nygard. ${ }^{12}$

\section{Statistical analysis}

Statistical analysis of changes in tremor amplitude was carried out using the Wilcoxon's test for paired differences. Analysis of changes in pulse rate was carried out by means of Student's $t$ test for paired data. Parameters determined during each active treatment period were compared with those determined after an equivalent period of placebo.

\section{Results}

\section{TREMOR FREQUENCY AND AMPLITUDE}

\section{DURING PLACEBO}

The frequency of the dominant peak of hand tremor in the patients included in the study ranged from $4 \cdot 2$ to $10.0 \mathrm{~Hz}$ (median $7 \cdot 2 \mathrm{~Hz}$ ). In any individual patient the peak frequency of tremor varied somewhat on the two occasions of recording but when all patients were considered the difference was not statistically significant. The magnitude of hand tremor at the dominant frequency ranged from 0.0013 to $0.236 \mathrm{~g}$ (median $0.0096 \mathrm{~g}$ ), which in terms of actual hand displacement corresponds to an amplitude of $0.006-$ $2.7 \mathrm{~mm}$ (median 0.062 mm).

Two patients had placebo discontinued after a period of two weeks because tremor interfered seriously with their daily activities. On the other hand, four patients had during placebo treatment tremor amplitude below $0.02 \mathrm{~mm}$, which is considered to be the upper limit of amplitude of physiological hand tremor in our laboratory (unpublished data). The same patients had consistently shown pathological values of tremor amplitude on several occasions before their recruitment in the study, an observation that underlines the marked placebo response in this type of disease.

\section{EFFECTS OF METOPROLOL AND PROPRANOLOL}

Tremor Only 13 patients received propranolol and metoprolol at the higher dosages (see below under Adverse Effects). Neither drug had any significant influence on the dominant peak frequency of tremor. At a dosage of $120 \mathrm{mg}$ daily, propranolol was found to be superior to placebo on the basis of the performance tests $(p<0.05)$ and patient's selfassessment ( $p<0 \cdot 01$ ). Propranolol $240 \mathrm{mg}$ daily was superior to placebo on accelerometric assessment 
$(p<0.02)$, performance tests $(p<0.01)$, clinical assessment $(\mathrm{p}<0.05)$ and patients' self-assessment $(p<0.01)$. The median reductions in tremor amplitude during propranolol treatment at the dosages of 120 and $240 \mathrm{mg}$ were $25 \%$ (NS) and $45 \%$ $(\mathrm{p}<0.02)$ respectively (comparison made with the placebo period). The reduction in tremor amplitude at the higher dosage did not differ from that observed at the lower dosage (fig 1).

There was a trend for the amplitude of tremor to be lower during metoprolol treatment as compared to placebo (median reductions $13 \%$ at the $150 \mathrm{mg}$ dosage and $32 \%$ at the $300 \mathrm{mg}$ dosage), but the effect was not statistically significant. At neither of the two dosages used did the effect of metoprolol differ from that of placebo, irrespective of the method of assessment (figs 1 and 2). When the reductions in tremor amplitude (expressed as absolute or percent change values) produced by metoprolol and propranolol at dosages having equipotent $\beta_{1}$ blocking activity were compared, no statistically significant difference could be found between the two drugs. However, propranolol at a dosage of $120 \mathrm{mg}$ daily was superior to metoprolol ( $150 \mathrm{mg}$ daily) on the basis of clinical assessment $(p<0.05)$ and patient's self assessment $(p<0.01)$. At the dosage of $240 \mathrm{mg}$ daily, propranolol was superior to metoprolol $(300$ $\mathrm{mg}$ daily) on the basis of patient's self-assessment $(p<0.05)$ (fig 2). Four patients on propranolol and three patients on metoprolol were found to have tremor amplitudes within the range of physiological tremor $(<0.02 \mathrm{~mm}$ hand displacement $)$.

When asked which of the three treatments they

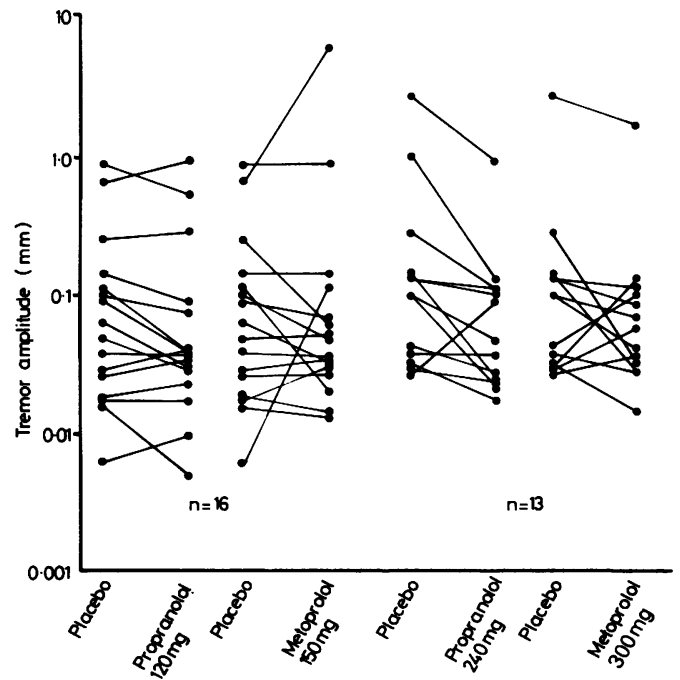

Fig 1 Effect of propranolol and metoprolol on tremor amplitude in the patients included in the study.

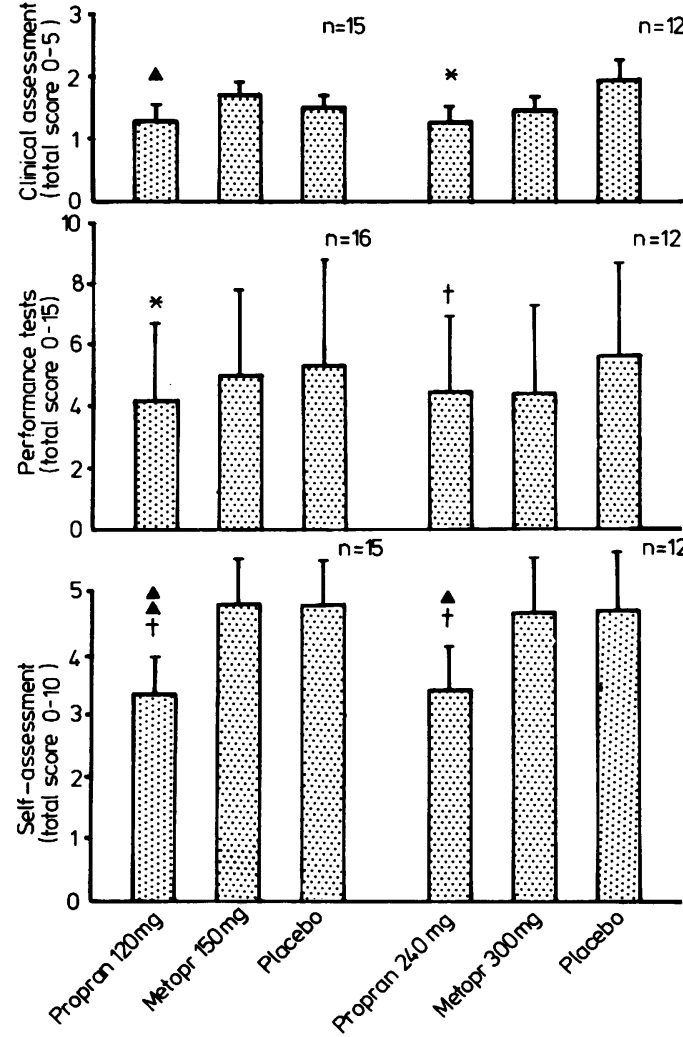

Fig 2 Mean score for clinical assessment, performance tests and patient's self-assessment during propranolol, metoprolol and placebo treatments.

Bars represent mean $\pm \operatorname{sem}\left({ }^{*} p<0.05\right.$ and ${ }^{\dagger} p<0.01$ as compared to placebo; $\Delta p<0.05$ and $\Delta p<0.01$ as compared to metoprolol).

considered best, six patients chose propranolol, two patients metoprolol and one patient placebo. Six patients could not find any difference between the three treatments, whereas one patient found propranolol as effective as placebo but superior to metoprolol.

Pulse rate The tachycardic response on standing was lower during active drug treatments than during placebo (fig 3). The difference, however, was statistically significant only at the higher dosages of both metoprolol and propranolol. The degree of inhibition of standing tachycardia was similar for the two drugs.

Serum propranolol and metoprolol levels Serum concentrations of propranolol were found to vary from 21.0 to $164.0 \mathrm{ng} / \mathrm{ml}$ (median $44.0 \mathrm{ng} / \mathrm{ml}$ ) and 


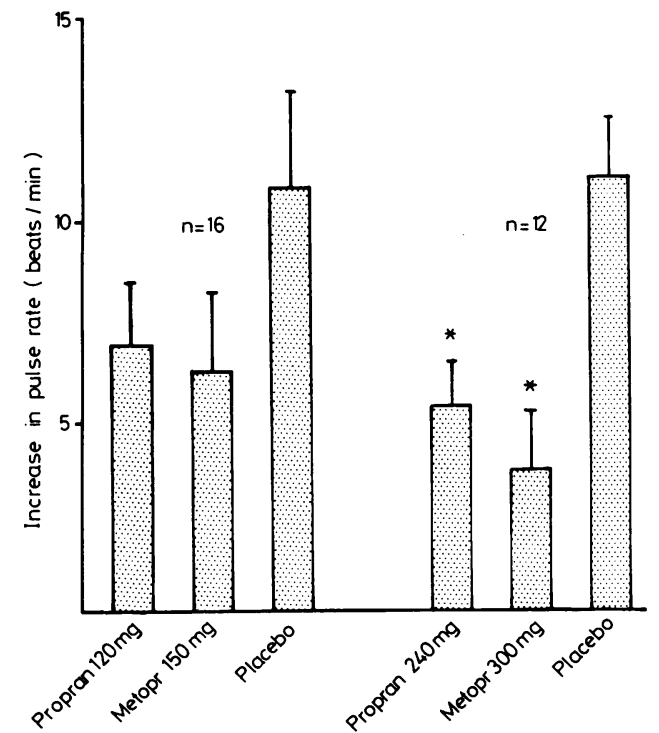

Fig 3 Effect of the various treatments on standing tachycardia. Bars represent the mean $\pm \operatorname{sem}\left({ }^{*} p<0.01\right.$ as compared to placebo).

from 60.0 to $296.0 \mathrm{ng} / \mathrm{ml}$ (median $113.1 \mathrm{ng} / \mathrm{ml}$ ) on daily dosage of $120 \mathrm{mg}$ and $240 \mathrm{mg}$ respectively. Serum metoprolol levels ranged from less than $5 \mathrm{ng} / \mathrm{ml}$ to $191.0 \mathrm{ng} / \mathrm{ml}($ median $80.0 \mathrm{ng} / \mathrm{ml}$ ) on the lower daily dosage and from less than $5 \mathrm{ng} / \mathrm{ml}$ to $555.6 \mathrm{ng} / \mathrm{ml}$ (median $206.8 \mathrm{ng} / \mathrm{ml}$ ) on the higher daily dosage of the drug.

Adverse effects Adverse effects were reported by an appreciable proportion of patients with the two active treatments and placebo. These effects are summarised in the table. Owing to the occurrence of breathlessness two patients received only the lower doses of both propranolol and metoprolol. A third patient, because of failure to attend the clinic,

Table Side effects

\begin{tabular}{llll}
\hline & \multicolumn{2}{l}{ Number of patients } \\
\cline { 2 - 4 } & Propranolol & Metoprolol & Placebo \\
\hline Tiredness & 5 & 3 & 2 \\
Headache & 2 & - & 1 \\
Vivid dreams & 2 & 1 & 2 \\
Loss of concentration & 1 & 2 & - \\
Breathlessness & 2 & 2 & - \\
Sedation, depression & 1 & 2 & - \\
Blurred vision & - & 1 & - \\
Feeling of unreality, & - & 1 & - \\
sleeplessness & - & - & - \\
Muscle pain on exercise & 1 & - & 2 \\
Anxiety, irritability & - & 1 & 1 \\
Sexual difficulty & 1 &
\end{tabular}

received only the lower dose of propranolol. In no case were side effects sufficiently severe to cause a patient's withdrawal from the study.

\section{Discussion}

The present study failed to provide a demonstration that metoprolol, at dosages as high as $\mathbf{3 0 0} \mathrm{mg}$ daily, is effective in the treatment of essential tremor. Although there was a tendency for our patients to do better on metoprolol than on placebo, the difference failed to reach statistical significance, irrespective of dosage or method of assessment. In contrast, propranolol at the dosage of $120 \mathrm{mg}$ daily was found to be superior to placebo on performance tests and patient's self assessment, whereas at the higher dosage ( $240 \mathrm{mg}$ daily) it was superior to placebo on all the methods of assessment used. When dosages having equivalent $\beta_{1}$-blocking activity were compared, propranolol proved to be significantly better than metoprolol on the basis of clinical assessment and patient's self-evaluation. Our results are in agreement with those of Leigh $e t a l$ who found metoprolol $(50 \mathrm{mg}$ twice daily) less effective than sotalol ( $80 \mathrm{mg}$ twice daily) and no better than placebo in reducing the severity of essential tremor in 17 patients. On the other hand, our results are partly in contrast with those reported by Larsen and Teravainen $^{8}$ who found metoprolol $(50 \mathrm{mg}$ three times daily) superior to placebo (even though less so than propranolol, $80 \mathrm{mg}$ three times daily) in reducing tremor amplitude in 24 patients.

The most interesting comparison can be made with the results of our previous study, in which single oral doses of metoprolol and propranolol ${ }^{9}$ were found to be better than placebo and virtually equipotent in the same population of patients. We cannot give any clear explanation for the apparent loss or reduction of efficacy of metoprolol during prolonged administration. It may be argued that it is usually more difficult to demonstrate a statistically significant drug effect when the evaluation of its pharmacodynamic action is made at long intervals of time and the symptom (essential tremor) is subject to considerable day-to-day variability. However, under the same experimental conditions and in the same patients it was possible to show a clearcut effect of propranolol at dosages that are considered to be equipotent in terms of $\beta_{1}$-blockade. A second possible explanation for the discrepancies between the results of the acute and of the chronic study may be related to differences in protocol. While in the acute study metoprolol was given as a single $150 \mathrm{mg}$ dose 90 minutes before tremor assessment, in the chronic study the drug was given in divided daily administrations so that the individual dose taken before the assessment was 
smaller (50 or $100 \mathrm{mg}$ ). Moreover, in the chronic study the interval between drug intake and time of assessment (usually 120-180 min) could not be so rigidly standardised. Since metoprolol is rapidly absorbed, has a short half-life and does not accumulate during repeated administration, it is possible that the serum concentration of the drug at the time of tremor assessment was greater during the acute study. Since serum metoprolol concentrations in that study were not measured, this possibility cannot be excluded. In this regard, it is noteworthy that serum propranolol concentrations in the range of that obtained following a single oral dose $(120 \mathrm{mg})^{13}$ were achieved with $240 \mathrm{mg}$ of the drug when administered chronically. However, since the pharmacokinetic differences between propranolol and metoprolol are not very marked, it is difficult to envisage how these could account for such a clear-cut differential response between these drugs in the two studies. Furthermore, the serum concentration of metoprolol (300 mg daily) at the time of assessment was sufficient to inhibit standing tachycardia to at least the same extent of that produced by the higher dosage of propranolol (fig 3 ).

Another possibility that needs to be considered is that tolerance to the tremorolytic properties of the two $\beta$-blockers developed. In the only controlled study ${ }^{8}$ in which metoprolol was found to be effective, the drug was, however, given for no longer than one week. In our own patients, there was a tendency for the response to propranolol to be less marked after prolonged administration as compared to that observed following a single oral $\operatorname{dose}^{9}$ (at dosages producing similar serum drug concentrations). It is possible, however, that during chronic administration the effect of propranolol was mediated by some additional mode of action, not shared by metoprolol and to which tolerance did not develop.

It has been suggested that $\beta_{1}$-selective adrenoceptor blocking drugs are less valuable than nonselective drugs in the treatment of essential tremor, ${ }^{14}$ but this view has been questioned. ${ }^{15}$ Since the cardioselectivity of metoprolol is at least partially lost at doses higher than $100-150 \mathrm{mg}^{16}$ the more favourable long-term response to propranolol in our patients cannot be taken as sound evidence that the tremorolytic effect was related to blockade of $\beta_{2}$ receptors.

Whatever the explanation for the disappointing response to prolonged administration of metoprolol, our results strongly suggest that in the absence of specific contraindications propranolol should be preferred to metoprolol in the treatment of essential tremor.
We would like to thank the physicians at the National Hospital, Queen Square, London, for allowing us to study patients under their care. We also thank the staff of the pharmacy at the National Hospital for their assistance. We are grateful to Dr J Ward, Dept of Clinical Pharmacology, Groby Road Hospital, Leicester, for measuring propranolol and metoprolol serum levels. SC has been supported by a European Science Foundation fellowship, European Training Programme in Brain and Behaviour Research 198081 (Strasbourg, France).

\section{References}

' Ljung O. Treatment of essential tremor with metoprolol. N Eng J Med 1979;301:1005.

2 Britt CW. Peters BH. Metoprolol for essential tremor. N Engl J Med 1979;301:331.

${ }^{3}$ Riley T, Fleet AB. Metoprolol tartrate for essential tremor. $N$ Engl J Med 1979;301:663.

+ Newman RP, Jacobs L. Metoprolol in essential tremor. Arch Neurol 1980;37:596-7.

5 Turnbull DM, Shaw DA. Metoprolol in essential tremor. Lancet 1980;i:95.

- Jefferson D, Jenner P, Marsden CD. $\beta$-adrenoceptor antagonists in essential tremor. $J$ Neurol Neurosurg Psychiatry 1979;42:904-9.

${ }^{7}$ Leigh PN, Marsden CD, Twomey A, Jefferson D. $\beta$-adrenoceptor antagonists and essential tremor. Lancet 1981 ;i:1106.

${ }^{\star}$ Larsen TA. Teravainen H. $\beta$-blockers in essential tremor. Lancet 1981 ;ii:533.

- Calzetti S, Findley LJ, Gresty MA, Perucca E, Richens A. Metoprolol and propranolol in essential tremor: a double-blind controlled study. J Neurol Neurosurg Psychiatry 1981;44:814-9.

10 Johnsson G, Nyberg G, Sölvell L. Influence of metoprolol and propranolol on hemodynamic effects induced by physical work and isoprenaline. Acta Pharmacol Toxicol (Kbh) 1975;36:69-75 (suppl V).

" Carruthers SG, Ghosal AG, McDevitt DG, Nelson JK, Shanks RG. The assessment of $\beta$-adrenoceptor blocking drugs in hyperthyroidism. $\mathrm{Br} J$ Clin Pharmacol 1974;1:93-8.

12 Nygard G, Shelver WM, Wahba Khalil SK. Sensitive high pressure liquid chromatographic determination of propranolol in plasma. J Pharm Sci 1979;68:379-81.

${ }^{13}$ Calzetti S, Findley LJ, Gresty MA, Perucca E, Richens A. The effect of an oral dose of propranolol on essential tremor. A double-blind controlled study. Am Neurol (in press).

it Beta-blockers in essential tremor (Editorial). Lancet 1979;ii: 1280.

15 Ljung O. Metoprolol in essential tremor. Lancet 1980; i: 1032 .

${ }^{16}$ Koch-Weser J. Metoprolol. N Engl J Med 1979; 301: 698-703. 